\title{
Current density maps, magnetizability, and nuclear magnetic shielding tensors of bis-heteropentalenes. II. Furo-furan Isomers
}

\author{
I. García Cuesta \\ Departament de Química Física, Institut de Ciència Molecular, Universitat de València, \\ Carrer Dr. Moliner 50, 46100 Burjassot, València, Spain
}

R. Soriano Jartín

Dipartimento di Chimica dell'Università degli Studi di Modena e Reggio Emilia, Via Campi 183, 41100 Modena, Italy

\author{
A. Sánchez de Merás \\ Departament de Química Física, Institut de Ciència Molecular, Universitat de València, \\ Carrer Dr. Moliner 50, 46100 Burjassot, València, Spain \\ P. Lazzeretti ${ }^{\mathrm{a})}$ \\ Dipartimento di Chimica dell'Università degli Studi di Modena e Reggio Emilia, Via Campi 183, \\ 41100 Modena, Italy
}

(Received 9 October 2003; accepted 5 January 2004)

\begin{abstract}
Magnetic susceptibility and nuclear magnetic shielding at the nuclei of bis-heteropentalenes formed by two furan units ([2,3- $b],[3,2-b],[3,4-b]$, and $[3,4-c]$ isomers) have been computed by several approximated techniques and a large Gaussian basis set to achieve near Hartree-Fock estimates. $A b$ initio models of the ring currents induced by a magnetic field normal to the molecular plane were obtained for the three isomeric systems of higher symmetry, showing that the $\pi$ electrons give rise to intense diamagnetic circulation. The $\pi$ currents are responsible for enhanced magnetic anisotropy and strong out-of-plane proton deshielding. The theoretical findings are used to build up a "diatropicity matrix" for two fused five-membered heterocyclic systems. () 2004 American Institute of Physics. [DOI: 10.1063/1.1650326]
\end{abstract}

\section{INTRODUCTION}

Several benchmarks of aromaticity have been proposed in the chemical literature. These topics constitute a matter of major debate within the community of theoretical organic chemists, who ask if a quantitative assessment of aromaticity is possible. ${ }^{1-4}$

Magnetic indicators have been chosen as preferential measures for aromaticity of fused hetero-bicycles, with the conclusion that the most aromatic systems are characterized by the least stable forms. ${ }^{5}$ The most stable positional isomers, for instance, $[3,2-b]$, need not have the largest aromatic or resonance stabilization energies.

As a matter of fact, molecules which contain two fused five-membered heterocyclic rings constitute an ideal "matrix" of compounds for comparative studies of diatropicity, that is, the ability of conjugated planar systems to sustain intense diamagnetic electronic currents in the presence of a magnetic field perpendicular to the molecular plane.

In the first paper of this series, ${ }^{6}$ hereafter referred to as I, four isomers of the dihydro-pyrrolo-pyrrole bicyclic system have been taken into account. They are placed in a "row" of the matrix, with the convention that diatropicity increases from left to right, according to the sequence

$$
[3,4-b] \rightarrow[2,3-b] \rightarrow[3,2-b] \rightarrow[3,4-c] .
$$

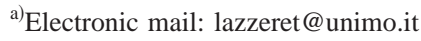

Other bicyclic compounds formed by five-membered heterocyclic molecules, referred to as bis-heteropentalenes, can be arranged in successive rows, according to the same criterion of increasing diatropicity. We expect that the "columns" of such matrix will contain bicyclic rings with the same isomeric structure and with different heteroatom. They can be ordered in a similar way, by diatropicity increasing from top to bottom of each column.

The ordering (1) corresponds to that of increasing absolute value of the out-of-plane component of the magnetic susceptibility tensor, which was adopted to quantify relative diatropicity, that is, "magnetic aromaticity." ${ }^{6}$ The different rows can therefore be ordered according to the same criterion.

This matrix could give explicit quantitative information, and possibly benchmarks of magnetotropicity/aromaticity to compare with other measures proposed in the literature. ${ }^{1-3}$

The present paper is aimed at evaluating magnetic susceptibility and nuclear magnetic shieldings in the series (1)(4) furo-furans via different procedures at the coupled Hartree-Fock level of accuracy. The theoretical results are discussed in relation to the magnetic aromaticity of these systems.

\section{COMPUTATIONAL DETAILS}

The Gaussian basis sets adopted in this study are the fully noncontracted $(13 s 8 p 4 d)$ for carbon, $(13 s 8 p 4 d 1 f)$ 
TABLE I. Bond lengths $(\AA)$.

\begin{tabular}{|c|c|c|c|c|c|c|c|}
\hline \multicolumn{4}{|c|}{$[3,4-b] C_{s}$ symmetry } & \multicolumn{4}{|c|}{$[3,2-b] C_{2 h}$ symmetry } \\
\hline $\mathrm{O} 1-\mathrm{C} 1$ & 1.370 & C1-H1 & 1.078 & $\mathrm{C} 1-\mathrm{O} 1$ & 1.382 & $\mathrm{C} 1-\mathrm{H} 1$ & 1.079 \\
\hline $\mathrm{C} 1-\mathrm{C} 2$ & 1.368 & $\mathrm{C} 5-\mathrm{H} 3$ & 1.081 & O1-C6 & 1.360 & $\mathrm{C} 3-\mathrm{H} 3$ & 1.080 \\
\hline $\mathrm{C} 2-\mathrm{C} 5$ & 1.448 & C6-H4 & 1.080 & C6-C5 & 1.367 & & \\
\hline C5-C6 & 1.355 & $\mathrm{C} 4-\mathrm{H} 2$ & 1.077 & $\mathrm{C} 5-\mathrm{C} 3$ & 1.425 & & \\
\hline $\mathrm{C} 6-\mathrm{O} 2$ & 1.387 & & & $\mathrm{C} 3-\mathrm{C} 1$ & 1.370 & & \\
\hline $\mathrm{O} 2-\mathrm{C} 3$ & 1.367 & & & & & & \\
\hline $\mathrm{C} 3-\mathrm{C} 4$ & 1.359 & & & & & & \\
\hline $\mathrm{C} 4-\mathrm{O} 1$ & 1.373 & & & & & & \\
\hline $\mathrm{C} 2-\mathrm{C} 3$ & 1.428 & & & & & & \\
\hline \multicolumn{4}{|c|}{$[2,3-b] C_{2 v}$ symmetry } & \multicolumn{4}{|c|}{$[3,4-c] D_{2 h}$ symmetry } \\
\hline $\mathrm{C} 1-\mathrm{O} 1$ & 1.397 & $\mathrm{C} 1-\mathrm{H} 1$ & 1.078 & $\mathrm{O} 1-\mathrm{C} 1$ & 1.354 & $\mathrm{C} 1-\mathrm{H} 1$ & 1.077 \\
\hline $\mathrm{O} 1-\mathrm{C} 6$ & 1.338 & C3-H3 & 1.081 & $\mathrm{C} 1-\mathrm{C} 5$ & 1.390 & & \\
\hline C6-C5 & 1.364 & & & C5-C6 & 1.468 & & \\
\hline $\mathrm{C} 5-\mathrm{C} 3$ & 1.443 & & & & & & \\
\hline $\mathrm{C} 3-\mathrm{C} 1$ & 1.364 & & & & & & \\
\hline
\end{tabular}

for oxygen, and $(8 s 3 p)$ for hydrogen. The $(s / p)$ substrata are from the van Duijneveldt tables, ${ }^{7}$ and the exponents of the polarization functions have been chosen to maximize paramagnetic susceptibilities in smaller size molecules. ${ }^{8}$

The $3 d$ exponents for carbon are 5.36, 1.44, 0.516, and 0.208 , the $3 d$ exponents for oxygen are $4.01,1.22,0.36$, and 0.10 , the $4 f$ exponent for oxygen is 0.94 . The $2 p$ exponents for hydrogen are 4.02, 0.952, and 0.294.

Molecular geometries have been optimized by the GAUSSIAN code, ${ }^{9}$ using the $6-31 \mathrm{G}^{*}$ basis set within the B3LYP approach. All the molecules are predicted to be planar. The optimized bond lengths are shown in Table I. They have been employed in the subsequent calculation of magnetic properties and current density maps via the SYSMO code. $^{10}$

Six different computational schemes were applied. The first five retain the gaugeless basis sets $(13 s 8 p 4 d 1 f / 13 s 8 p 4 d / 8 s 3 p)$ for $\mathrm{O} / \mathrm{C} / \mathrm{H}$. They are the conventional common origin $(\mathrm{CO})$ approach and four proce- dures, CTOCD-DZ1, CTOCD-DZ2, CTOCD-PZ1, and CTOCD-PZ2, relying on continuous transformation of the origin of the current density (CTOCD), whereby either the diamagnetic (DZ) or the paramagnetic (PZ) contributions are formally set to zero. ${ }^{11-14}$

Whereas DZ1 and DZ2 schemes are analytical, the DZ2 and PZ2 variants, introducing damping factors to improve the accuracy of computed magnetic shielding, are based on numerical integration. Magnetic susceptibilities from DZ1 and DZ2 (PZ1 and PZ2) are the same to several significant figures. The theory of CTOCD methods can be found elsewhere. ${ }^{1,15-17}$

The approach based on London orbitals (LO), ${ }^{18,19}$ sometimes referred to as gauge-including atomic orbitals (GIAO), implemented in the DALTON package, ${ }^{20}$ has also been used. The basis sets are again $(13 s 8 p 4 d)$ for carbon, $(13 s 8 p 4 d 1 f)$ for oxygen, and $(8 s 3 p)$ for hydrogen, but the Gaussian orbitals include gauge factors à la London.

TABLE II. Magnetic susceptibility tensor of furo-furans in (cgs) ppm a.u. ${ }^{a}$ (origin in the center of mass). Anisotropy $\Delta \chi=\chi_{z z}-\frac{1}{2}\left(\chi_{x x}+\chi_{y y}\right)$.

\begin{tabular}{lcccccc}
\hline \hline & $x x$ & $y y$ & $z z$ & $x y$ & $a v$ & $\Delta \chi$ \\
\hline$[3,4-b]$ & & & & & & \\
$\chi^{d}+\chi^{p}(\mathrm{LO})$ & -511.69 & -532.62 & -1122.96 & -9.32 & -722.42 & -600.80 \\
{$[2,3-b]$} & & & & & & \\
$\chi^{\Delta}+\chi^{p}$ & -553.77 & -482.81 & -1176.42 & 0.00 & -737.67 & -658.12 \\
$\chi^{d}+\chi^{\Pi}$ & -561.42 & -497.90 & -1175.59 & 0.00 & -744.97 & -645.93 \\
$\chi^{d}+\chi^{p}(\mathrm{LO})$ & -561.83 & -506.73 & -1178.77 & 0.00 & -749.11 & -644.49 \\
{$[3,2-b]$} & & & & & & \\
$\chi^{\Delta}+\chi^{p}$ & -538.38 & -471.30 & -1197.67 & 24.79 & -735.78 & -692.83 \\
$\chi^{d}+\chi^{\Pi}$ & -546.38 & -484.57 & -1196.46 & 19.38 & -742.47 & -680.99 \\
$\chi^{d}+\chi^{p}(\mathrm{LO})$ & -546.47 & -492.86 & -1199.64 & 17.75 & -746.32 & -679.97 \\
{$[3,4-c]$} & & & & & & \\
$\chi^{\Delta}+\chi^{p}$ & -362.99 & -512.97 & -1417.85 & 0.00 & -764.60 & -979.87 \\
$\chi^{d}+\chi^{\Pi}$ & -379.10 & -513.27 & -1419.06 & 0.00 & -770.48 & -972.88 \\
$\chi^{d}+\chi^{p}(\mathrm{LO})$ & -390.52 & -505.12 & -1409.25 & 0.00 & -768.29 & -961.43 \\
\hline \hline
\end{tabular}

The conversion factor from cgs a.u. per molecule to cgs emu per mole is $a_{0}^{3} N_{A}=8.9238878 \times 10^{-2}$; further conversion to SI units is obtained by $1 \mathrm{JT}^{-2}=0.1 \mathrm{cgs}$ emu. 
TABLE III. Nuclear magnetic shielding in furo[2,3- $b]$ furan (in ppm).

\begin{tabular}{|c|c|c|c|c|c|}
\hline & $x x$ & $y y$ & $z z$ & $x y$ & $a v$ \\
\hline \multicolumn{6}{|l|}{$\mathrm{O} 1$} \\
\hline$\sigma^{\mathrm{DZ2}}$ & 0.86 & 31.83 & 266.95 & 31.82 & 99.88 \\
\hline$\sigma^{\mathrm{PZ} 2}$ & 1.15 & 32.19 & 266.93 & 31.81 & 100.09 \\
\hline$\sigma^{d}+\sigma^{p}(\mathrm{LO})$ & 1.19 & 32.11 & 266.81 & 32.14 & 100.04 \\
\hline \multicolumn{6}{|l|}{$\mathrm{C} 1$} \\
\hline$\sigma^{\mathrm{DZ2}}$ & -30.15 & 56.23 & 103.67 & 30.34 & 43.25 \\
\hline$\sigma^{\mathrm{PZ} 2}$ & -30.38 & 56.22 & 103.33 & 29.97 & 43.06 \\
\hline$\sigma^{d}+\sigma^{p}(\mathrm{LO})$ & -29.98 & 57.08 & 103.88 & 30.35 & 43.66 \\
\hline \multicolumn{6}{|l|}{$\mathrm{C} 3$} \\
\hline$\sigma^{\mathrm{DZ2}}$ & 14.99 & 39.11 & 170.09 & 53.82 & 74.73 \\
\hline$\sigma^{\mathrm{PZ} 2}$ & 14.86 & 39.19 & 169.95 & 53.84 & 74.67 \\
\hline$\sigma^{d}+\sigma^{p}(\mathrm{LO})$ & 15.19 & 39.52 & 170.31 & 54.15 & 75.01 \\
\hline \multicolumn{6}{|l|}{$\mathrm{C} 5$} \\
\hline$\sigma^{\mathrm{DZ2}}$ & 17.91 & 76.18 & 164.97 & 0.00 & 86.35 \\
\hline$\sigma^{\mathrm{PZ} 2}$ & 17.90 & 76.21 & 164.89 & 0.00 & 86.33 \\
\hline$\sigma^{d}+\sigma^{p}(\mathrm{LO})$ & 18.06 & 76.52 & 165.13 & 0.00 & 86.57 \\
\hline \multicolumn{6}{|l|}{ C6 } \\
\hline$\sigma^{\mathrm{DZ2}}$ & -65.52 & 32.13 & 78.96 & 0.00 & 15.19 \\
\hline$\sigma^{\mathrm{PZ} 2}$ & -65.77 & 31.67 & 78.43 & 0.00 & 14.78 \\
\hline$\sigma^{d}+\sigma^{p}(\mathrm{LO})$ & -65.20 & 32.98 & 79.13 & 0.00 & 15.64 \\
\hline \multicolumn{6}{|l|}{ H1 } \\
\hline$\sigma^{\mathrm{DZ2}}$ & 26.11 & 26.04 & 20.60 & -0.18 & 24.25 \\
\hline$\sigma^{\mathrm{PZ} 2}$ & 26.28 & 26.21 & 20.65 & -0.29 & 24.38 \\
\hline$\sigma^{d}+\sigma^{p}(\mathrm{LO})$ & 26.39 & 26.41 & 20.81 & -0.38 & 24.54 \\
\hline \multicolumn{6}{|l|}{ H3 } \\
\hline$\sigma^{\mathrm{DZ2}}$ & 25.61 & 26.40 & 22.04 & 0.07 & 24.68 \\
\hline$\sigma^{\mathrm{PZ} 2}$ & 25.67 & 26.63 & 22.13 & 0.04 & 24.81 \\
\hline$\sigma^{d}+\sigma^{p}(\mathrm{LO})$ & 25.92 & 26.78 & 22.34 & 0.19 & 25.01 \\
\hline
\end{tabular}

\section{A. Magnetic susceptibilities}

Theoretical predictions for magnetic susceptibility, in ppm (cgs) atomic units, are displayed in Table II. The comparatively less accurate $\mathrm{CO}$ results from the gaugeless $(13 s 8 p 4 d 1 f / 13 s 8 p 4 d / 8 s 3 p)$ basis set are not shown. The gauge invariant results obtained via the London orbitals, indicated by LO in Table II, are partitioned into diamagnetic, $\chi^{d}$, and paramagnetic, $\chi^{p}$, contributions, evaluated with respect to the center of mass.

The notation adopted for the various CTOCD estimates $^{15-17,21-24}$ is the same as in paper I of this series. ${ }^{6}$ The least symmetric molecule, furo[3,4- $b]$ furan, has been studied only via the GIAO approach of the DALTON package. ${ }^{20}$

The CTOCD-DZ and CTOCD-PZ values for the magnetic susceptibility of the $[3,2-b]$ and $[3,4-c]$ isomers, which have vanishing electric dipole moment, do not change in a gauge translation, whereas those of the $[3,4-b]$ and [2,3- $b]$ systems, formally dependent on the coordinate system adopted in the calculation, are practically invariant thanks to the high quality of the basis set used in the calculation of magnetic properties. (See Diagram.)

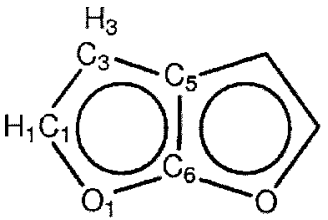

(1) $[2,3-b]$

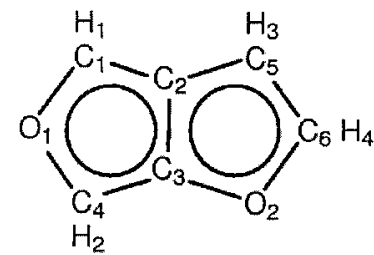

(3) $[3,4-b]$

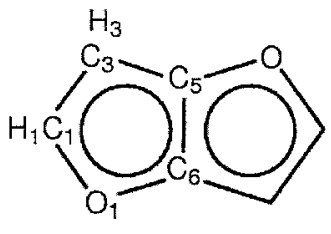

(2) $[3,2-b]$

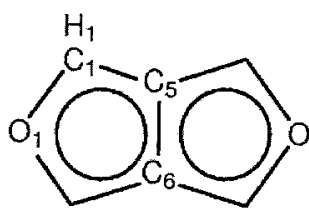

(4) $[3,4-c]$
The dipole velocity Thomas-Reiche-Kuhn sum rule is a condition for origin independence and charge conservation. ${ }^{1}$ For systems (1), (2), and (4), the average computed value is $\approx 55.8$, that is, $99.6 \%$ of the total number of electrons, 56 , confirming the excellent quality of the calculation.

The Pascal's rules indicate that, for a given $\mathrm{X}$, the four possible fused bicycles should have the same average magnetic susceptibility. Nonetheless, for $\mathrm{X}=\mathrm{O}$, the average in-plane LO susceptibility of 4 is $\approx-4.5 \times 10^{2}$ $\mathrm{ppm}$ (cgs) a.u., whereas the value for $\mathbf{1}-\mathbf{3}$ is $\approx-5.2$ $\times 10^{2} \mathrm{ppm}(\mathrm{cgs})$ a.u. A similar trend was observed in dihydro pyrrolo-pyrroles, ${ }^{6}$ in which the average in-plane LO susceptibility of $\mathbf{4}$ is $\approx-5.7 \times 10^{2} \mathrm{ppm}$ (cgs) a.u., whereas the value for $1-3$ is $\approx-6.1 \times 10^{2} \mathrm{ppm}$ (cgs) a.u.

TABLE IV. Nuclear magnetic shielding in furo[3,2- $b]$ furan (in ppm).

\begin{tabular}{|c|c|c|c|c|c|}
\hline & $x x$ & $y y$ & $z z$ & $x y$ & $a v$ \\
\hline \multicolumn{6}{|l|}{$\mathrm{O} 1$} \\
\hline$\sigma^{\mathrm{DZ2}}$ & -17.51 & 44.75 & 302.77 & -12.04 & 110.00 \\
\hline$\sigma^{\mathrm{PZ} 2}$ & -17.21 & 45.17 & 302.76 & -11.95 & 110.24 \\
\hline$\sigma^{d}+\sigma^{p}(\mathrm{LO})$ & -16.51 & 45.39 & 302.79 & -11.88 & 110.56 \\
\hline \multicolumn{6}{|l|}{$\mathrm{C} 1$} \\
\hline$\sigma^{\mathrm{DZ2}}$ & -34.65 & 32.55 & 105.91 & 43.74 & 34.60 \\
\hline$\sigma^{\mathrm{PZ2}}$ & -34.98 & 32.55 & 105.56 & 43.72 & 34.38 \\
\hline$\sigma^{d}+\sigma^{p}(\mathrm{LO})$ & -34.45 & 33.31 & 105.95 & 43.96 & 34.94 \\
\hline \multicolumn{6}{|l|}{$\mathrm{C} 3$} \\
\hline$\sigma^{\mathrm{DZ} 2}$ & 36.93 & 40.31 & 171.57 & 50.68 & 82.94 \\
\hline$\sigma^{\mathrm{PZ} 2}$ & 36.82 & 40.40 & 171.45 & 50.83 & 82.89 \\
\hline$\sigma^{d}+\sigma^{p}(\mathrm{LO})$ & 37.24 & 40.54 & 171.65 & 50.90 & 83.14 \\
\hline \multicolumn{6}{|l|}{ C5 } \\
\hline$\sigma^{\mathrm{DZ2}}$ & -40.02 & 33.45 & 110.88 & -4.57 & 34.77 \\
\hline$\sigma^{\mathrm{PZ} 2}$ & -40.15 & 33.14 & 110.60 & -4.40 & 34.53 \\
\hline$\sigma^{d}+\sigma^{p}(\mathrm{LO})$ & -39.90 & 34.05 & 110.85 & -4.67 & 35.00 \\
\hline \multicolumn{6}{|l|}{$\mathrm{H} 1$} \\
\hline$\sigma^{\mathrm{DZ2}}$ & 25.51 & 25.88 & 20.41 & -0.74 & 23.93 \\
\hline$\sigma^{\mathrm{PZ} 2}$ & 25.67 & 26.06 & 20.46 & -0.69 & 24.07 \\
\hline$\sigma^{d}+\sigma^{p}(\mathrm{LO})$ & 25.79 & 26.25 & 20.59 & -0.65 & 24.21 \\
\hline \multicolumn{6}{|l|}{ H3 } \\
\hline$\sigma^{\mathrm{DZ2}}$ & 25.65 & 26.42 & 21.90 & 0.59 & 24.66 \\
\hline$\sigma^{\mathrm{PZ} 2}$ & 25.75 & 26.64 & 22.00 & 0.59 & 24.80 \\
\hline$\sigma^{d}+\sigma^{p}(\mathrm{LO})$ & 26.02 & 26.76 & 22.19 & 0.64 & 24.99 \\
\hline
\end{tabular}


TABLE V. Nuclear magnetic shielding in furo[3,4- $b]$ furan (in ppm) via GIAO basis set.

\begin{tabular}{lccccc}
\hline \hline & $x x$ & $y y$ & $z z$ & $x y$ & $a v$ \\
\hline $\mathrm{O} 1$ & -16.61 & 9.47 & 229.96 & -23.87 & 74.27 \\
$\sigma^{d}+\sigma^{p}(\mathrm{LO})$ & & & & & \\
$\mathrm{C} 1$ & & & & & \\
$\sigma^{d}+\sigma^{p}(\mathrm{LO})$ & 44.17 & -11.05 & 125.26 & -12.42 & 52.79 \\
$\mathrm{C} 2$ & & & & & \\
$\sigma^{d}+\sigma^{p}(\mathrm{LO})$ & 42.21 & -6.32 & 149.88 & 36.20 & 61.92 \\
$\mathrm{C} 3$ & & & & & \\
$\sigma^{d}+\sigma^{p}(\mathrm{LO})$ & 16.12 & -20.34 & 99.82 & -50.96 & 31.87 \\
$\mathrm{C} 4$ & & & & & \\
$\sigma^{d}+\sigma^{p}(\mathrm{LO})$ & 50.17 & 12.85 & 130.02 & 18.88 & 64.35 \\
$\mathrm{C} 5$ & 24.99 & 55.51 & 169.13 & 57.75 & 83.21 \\
$\sigma^{d}+\sigma^{p}(\mathrm{LO})$ & & & & & \\
$\mathrm{C} 6$ & & & & & \\
$\sigma^{d}+\sigma^{p}(\mathrm{LO})$ & -46.46 & 33.65 & 96.04 & 75.83 & 27.74 \\
$\mathrm{H} 1$ & 25.83 & 26.99 & 20.45 & -0.37 & 24.42 \\
$\sigma^{d}+\sigma^{p}(\mathrm{LO})$ & & & & & \\
$\mathrm{H} 2$ & 25.88 & 26.45 & 20.61 & 0.44 & 24.31 \\
$\sigma^{d}+\sigma^{p}(\mathrm{LO})$ & & & & & \\
$\mathrm{H} 3$ & 25.82 & 27.39 & 22.71 & 0.35 & 25.31 \\
$\sigma^{d}+\sigma^{p}(\mathrm{LO})$ & & & & & \\
$\mathrm{H} 4$ & 25.58 & 26.55 & 21.18 & 1.72 & 24.44 \\
$\sigma^{d}+\sigma^{p}(\mathrm{LO})$ & & & & & \\
\hline \hline
\end{tabular}

A tentative explanation for these results may be attempted in terms of $\mathrm{C}-\mathrm{C}$ bond lengths in different isomers. Sequences of approximately single and double peripheral bonds are observed for 1-3 molecules, whereas bond length equalization $(\approx 1.40 \AA)$, consistent with $D_{2 h}$ symmetry, characterizes the $\mathbf{4}$ system, in which $\pi$-electron delocalization is higher. Further investigations on other bis-heteropentalene compounds are however necessary to understand this point in relation to magnetic aromaticity, which is usually ascribed only to the special behavior of $\pi$-electron clouds in the presence of magnetic field perpendicular to the molecular plane of aromatics. ${ }^{1-4}$

In fact the largest deviations from the systematic of Pascal are observed for the out-of-plane susceptibility, which is significantly biased by ring currents. Actually, the in-plane components are predicted (by all CTOCD and by GIAO methods) from two to three times smaller, in absolute value, than the out-of-plane component. This suggests that, relying on magnetic criteria as proposed in Ref. 5, furo-furans can be considered aromatic molecules.

Nonetheless, the theoretical results for the in-plane components have not fully converged. The highest discrepancies are observed in Table II for the $x x$ component of the [3,4-c] isomer, with $x$ the longitudinal axis of the molecule. The CTOCD and LO predictions for $\chi_{x x}$ lie in the quite large interval $-\approx 363-\approx 391$ (cgs) ppm a.u. Despite its big size, the basis set does not seem to have sufficient flexibility to describe charge rotation about the $x$ axis.

On the other hand, very good agreement was found among LO, DZ, and PZ predictions for the out-of-plane component, $\chi_{z z}$, of the susceptibility, for all four isomers. The differences are $\approx 0.3 \%-0.7 \%$, which means that the estimates are of near Hartree-Fock quality and can be used for measuring relative magnetic aromaticities.
The diatropicity scale of the furo-furan isomers, based on increasing absolute value of $\chi_{z z}$, is the same as that predicted for dihydro-pyrrolo-pyrroles, ${ }^{6}$ corresponding to Eq. (1).

The absolute value of the theoretical $\chi_{z z}$ of a given isomer of the furo-furan series is smaller than that of the corresponding dihydro-pyrrolo-pyrrole, ${ }^{6}$ compare, for instance, the LO predictions for the [3,4-c] isomer, -1409.25 in Table II of this paper with $-1623.69 \mathrm{ppm}$ a.u. in Table II of Ref. 6.

If, according to the prescription of Sec. I, we convene to place the corresponding isomers of the series $\mathrm{C}_{6} \mathrm{H}_{4} \mathrm{X}$, for $\mathrm{X}=\mathrm{NH}, \mathrm{O}, \mathrm{S}, \mathrm{P}, \ldots$, along the columns of the matrix, in increasing absolute value of $\chi_{z z}$, then the row for $\mathrm{X}=\mathrm{O}$ precedes that for $\mathrm{X}=\mathrm{NH}$.

Magnetic anisotropies $\Delta \chi=\chi_{z z}-(1 / 2)\left(\chi_{x x}+\chi_{y y}\right)$ have been proposed by Flygare and co-workers as fundamental quantitative descriptors of aromaticity. ${ }^{25,26}$ Theoretical $\Delta \chi$ results of Table II are enhanced and increase in absolute value in the same direction as $\left|\chi_{z z}\right|$, evidencing diatropicity of the furo-furans.

However, $|\Delta \chi|$ of a given furo-furan isomer is smaller than that of the corresponding pyrrolo-pyrrole. Moreover, only $\Delta \chi$ of the [3,4-c] isomer is bigger in absolute value than the experimental value of benzene, $-669.1 \pm 1(\mathrm{cgs})$ ppm a.u. ${ }^{27}$

\section{B. Nuclear magnetic shieldings}

Theoretical predictions of nuclear magnetic shieldings from CTOCD and LO methods are reported in Tables III-VI. Slowly converging CO predictions are not displayed. DZ2, PZ2, and LO magnetic shieldings of hydrogen, carbon, and oxygen nuclei in (1), (2), and (4) are very close to each other, or virtually the same in most cases. We conclude that (i) near Hartree-Fock accuracy has been achieved in the calculation, (ii) gaugeless DZ2 and PZ2 schemes are competitive with those based on London orbitals for the calculation of nuclear magnetic shieldings.

TABLE VI. Nuclear magnetic shielding in furo[3,4-c] furan (in ppm).

\begin{tabular}{lccccc}
\hline \hline & $x x$ & $y y$ & $z z$ & $x y$ & $a v$ \\
\hline $\mathrm{O} 1$ & & & & & \\
$\sigma^{\mathrm{DZ2}}$ & -220.77 & -218.83 & 232.72 & 0.00 & -68.96 \\
$\sigma^{\mathrm{PZ2}}$ & -220.21 & -218.57 & 232.68 & 0.00 & -68.70 \\
$\sigma^{d}+\sigma^{p}(\mathrm{LO})$ & -219.76 & -218.96 & 232.93 & 0.00 & -68.60 \\
$\mathrm{C} 1$ & & & & & \\
$\sigma^{\mathrm{DZ2}}$ & 20.27 & 12.18 & 127.09 & -27.96 & 53.18 \\
$\sigma^{\mathrm{PZ2}}$ & 20.26 & 12.06 & 126.79 & -28.27 & 53.04 \\
$\sigma^{d}+\sigma^{p}(\mathrm{LO})$ & 21.03 & 12.16 & 127.07 & -27.79 & 53.42 \\
$\mathrm{C}^{\mathrm{DZ2}}$ & & & & & \\
$\sigma^{\mathrm{PZ2}}$ & 48.97 & -27.54 & 152.40 & 0.00 & 57.94 \\
$\sigma^{d}+\sigma^{p}(\mathrm{LO})$ & 49.06 & -27.88 & 152.28 & 0.00 & 57.82 \\
$\mathrm{H} 1$ & 49.58 & -27.86 & 152.17 & 0.00 & 57.96 \\
$\sigma^{\mathrm{DZ2}}$ & & & & & \\
$\sigma^{\mathrm{PZ2}}$ & 23.91 & 26.13 & 18.83 & -0.36 & 22.96 \\
$\sigma^{d}+\sigma^{p}(\mathrm{LO})$ & 24.06 & 26.28 & 18.91 & -0.44 & 23.09 \\
\hline \hline
\end{tabular}



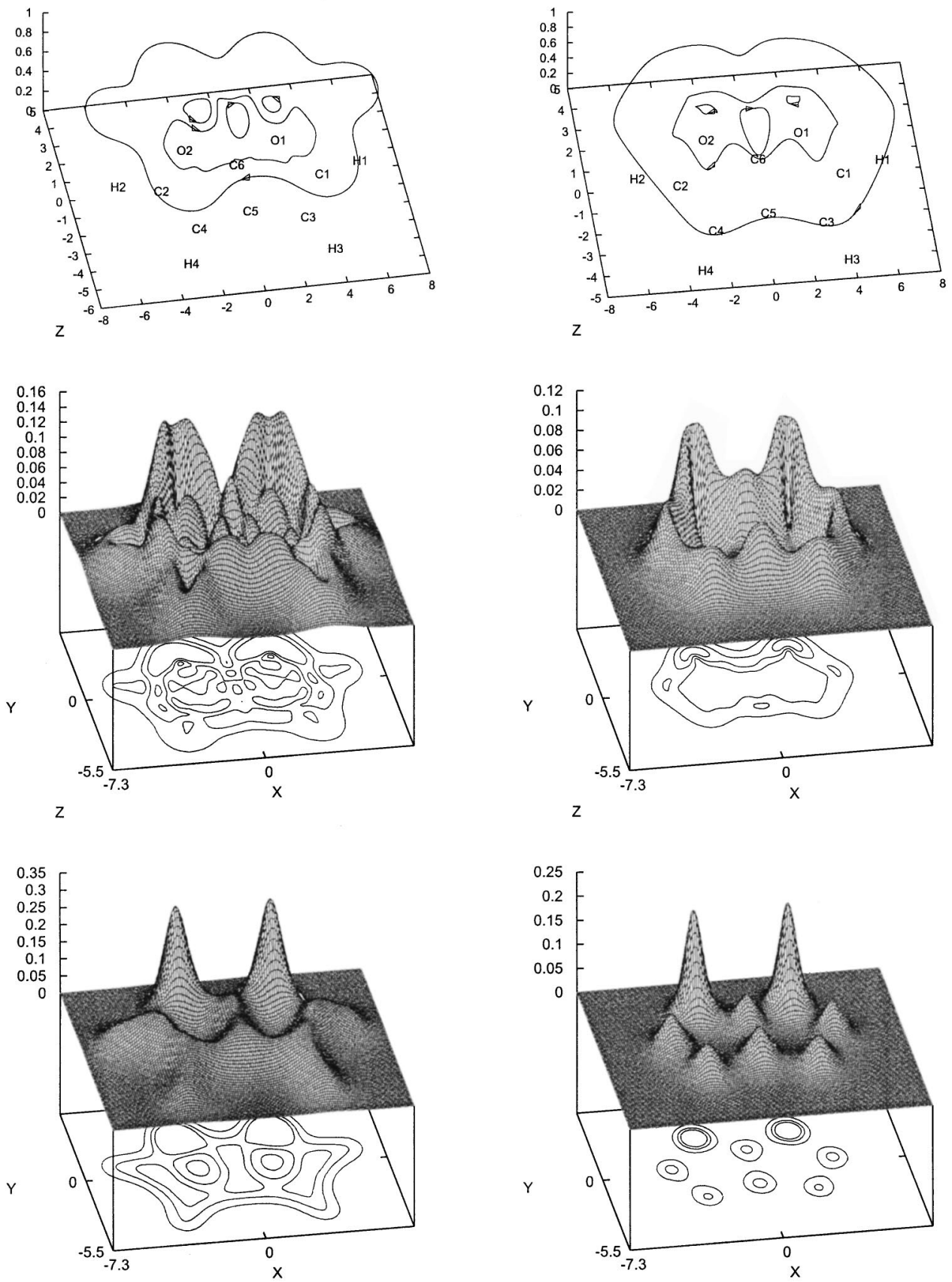

FIG. 1. The ring-current model for furo[2,3- $b]$ furan (1). From top to bottom, representations of the streamlines of the current density vector field $\mathbf{J}$ induced in the region of maximum $\pi$ electron density by a magnetic field of unit magnitude along the positive $z$ direction at right angles to the molecular plane, of its modulus, $|\mathbf{J}|$, and of $\rho$, the unperturbed electron charge density (in a.u.). Diamagnetic circulation is clockwise. The plots display a three-dimensional perspective view at points in planes parallel to the plane of the molecule, displaced from it by 0.8 bohr. Corresponding contour maps are shown with values $0.02 n, n=0,1,2, \ldots$ for $|\mathbf{J}|$, and $0.05 n, n=0,1,2, \ldots$ for the charge density. The maps on the left are relative to the total electronic distribution, those on the right are for the $\pi$-electron contribution.

It has long been known that proton magnetic shieldings in aromatic systems show a typical down-field (paramagnetic) shift of the out-of-plane component. Such shifts are usually explained within the framework of the ring-current model (RCM) in terms of enforced magnetic field at right angles to the molecular plane in the vicinity of hydrogen nuclei. ${ }^{1,28}$ Hydrogen magnetic shieldings are used for measuring aromaticity of planar unsaturated systems, see, for instance, a recent review article by Mitchell. ${ }^{28}$

The theoretical out-of-plane components reported in
Tables III-VI for (1), (2), and (3) are as large as $\approx 20-22$ ppm (the theoretical value for benzene is $20.65 \mathrm{ppm}$, see Table VI of Ref. 29), compared with $\approx 24-26 \mathrm{ppm}$ for the in-plane components.

It can be observed that a "diatropicity matrix" fully consistent with that built up in terms of out-of-plane susceptibility, see Sec. II A, is obtained adopting as a measure the outof-plane shielding of the proton in position 3 with respect to the heteroatom in the same ring. The same trend is found within each of DZ2, PZ2, and LO sets of data for (1), (2), 

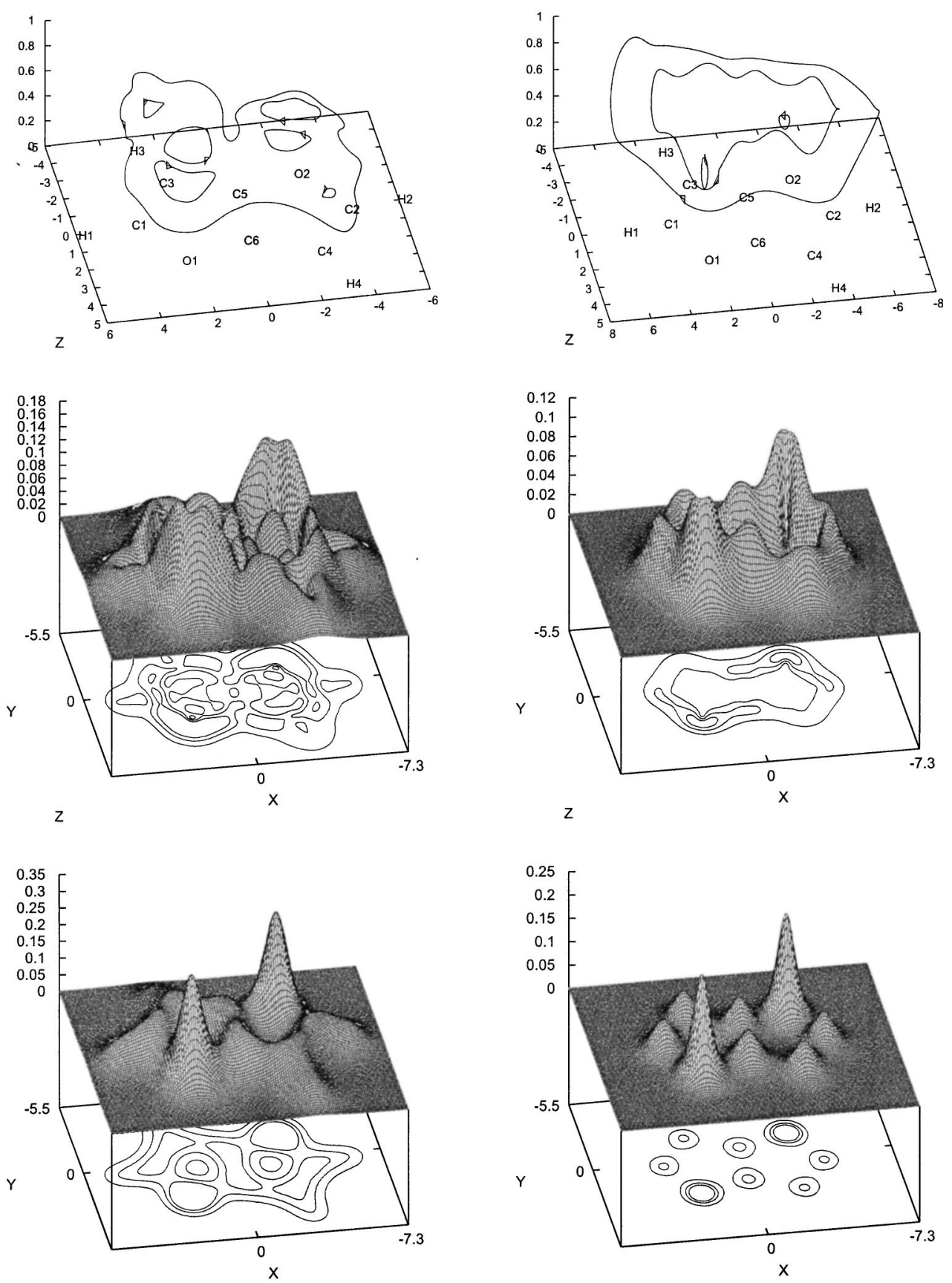

FIG. 2. The ring-current model for furo[3,2-b]furan (2). The conventions are the same as in Fig. 1.

and (3) systems. The out-of-plane shielding decreases along the rows and the columns of the matrix, in the direction of increasing diatropicity.

The largest paramagnetic shift was predicted for proton shielding in the $[3,4-c]$ isomer. Its perpendicular component is $\approx 19 \mathrm{ppm}$, that is, $\approx 1 \mathrm{ppm}$ smaller than that of benzene protons. An analogous result was found for the corresponding nitrogen-containing bis-heteropentalene, in which part of deshielding was attributed to the neighbor nitrogen atom. ${ }^{6}$ An analogous mechanism may be at work in the $[3,4-c]$ furo-furan

The $\pi$ ring currents could not play any special role in the magnetic shielding of carbon and oxygen nuclei in the molecular skeleton of furo-furans, since the ring currents pass essentially over them. However, the most shielded $\sigma$ component is invariably that out-of-plane.

\section{VISUALIZATION OF DIATROPICITY BY CURRENT DENSITY MAPS}

Maps obtained via the CTOCD-DZ CHF procedure, describing the current density induced in the electron cloud of furo[2,3- $b]$ furan, furo[3,2- $b]$ furan, and furo[3,4-c] furan by a spatially uniform magnetic field $B$ directed perpendicular to the molecular plane, along the positive $z$ axis, are shown in Figs. 1-4. The molecular domain investigated here is that 

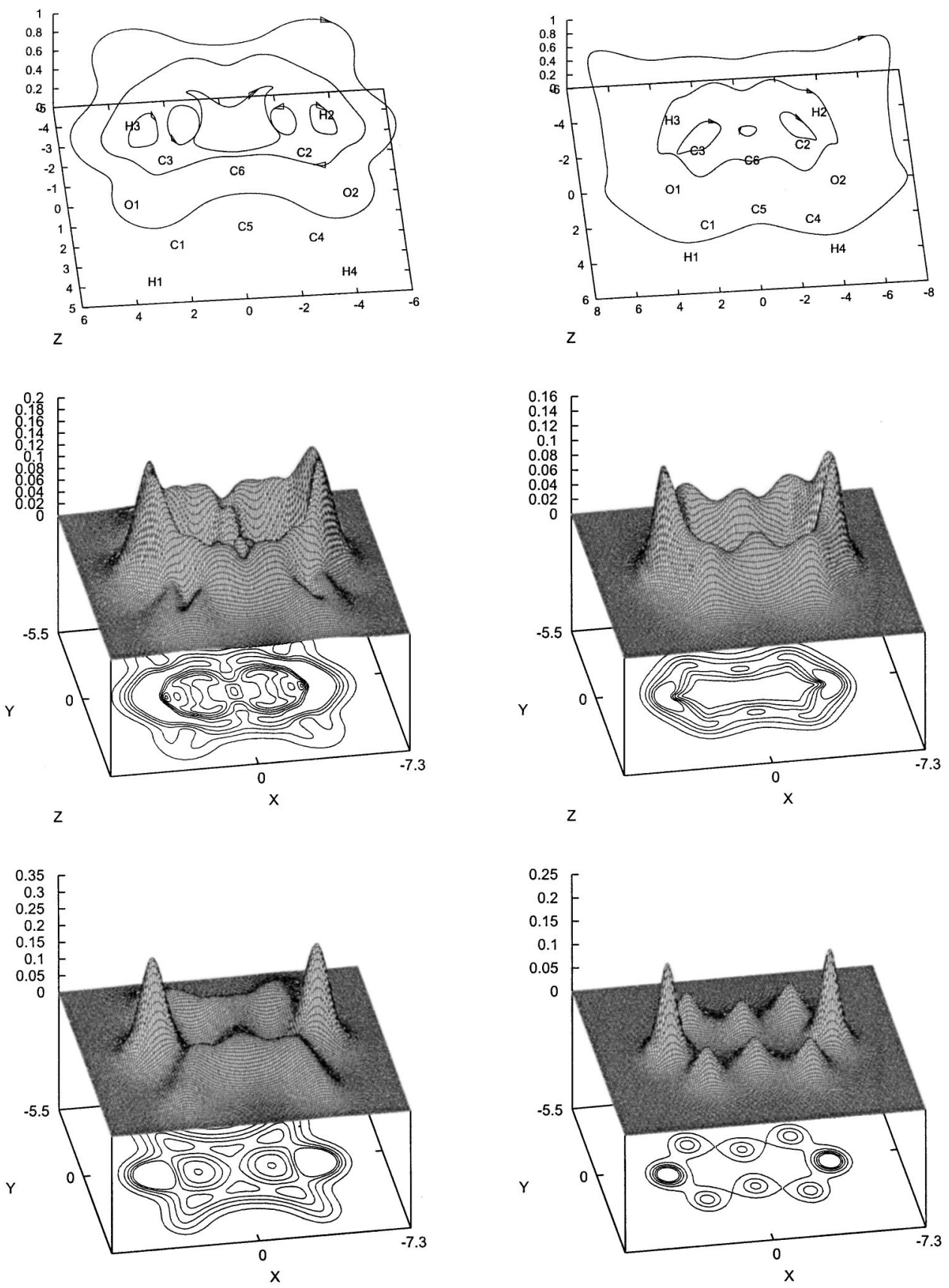

FIG. 3. The ring-current model for furo[3,4-c] furan (4). The conventions are the same as in Fig. 1.

of maximum $\pi$ density at about 0.8 bohr above the plane of the nuclei. Diamagnetic (paramagnetic) currents are clockwise (counterclockwise).

A limited number of streamlines accounting for the salient features of electron circulation is displayed for each molecule on top of Figs. 1-3. They provide fundamental information on the diatropic character of these compounds. They also document the oscillatory (leap-frog) effect ${ }^{30}$ of the $\pi$ ring currents, which are characterized by a considerable paramagnetic component in the domain investigated. A more refined representation for the streamlines on the plane at 0.8 bohr is displayed in Fig. 4.

The similarities with the maps reported for nitrogencontaining bis-heteropentalenes ${ }^{6}$ are evident. An almost iden- tical set of $\pi$ electron orbits, completely delocalized and surrounding the entire molecule, is observed. These figures provide the ring-current model for furo-furans and visualize the diatropicity of these compounds.

Three internal diamagnetic vortices can be observed in the $\pi$ current density of the $[2,3-b]$ and $[3,4-c]$ isomers in Fig. 4. In the latter they are aligned along the longitudinal axis. In the former two vortices rotate in the proximity of the oxygen atoms, and two foci are found on the opposite sides of the central vortex. The streamlines spiral in (out) about the right (left) focus.

The orbits for the total current density field, shown on the top left of Figs. 1-3, and on the left of Fig. 4, reveal the complicated topology of the field. The maps show that the 

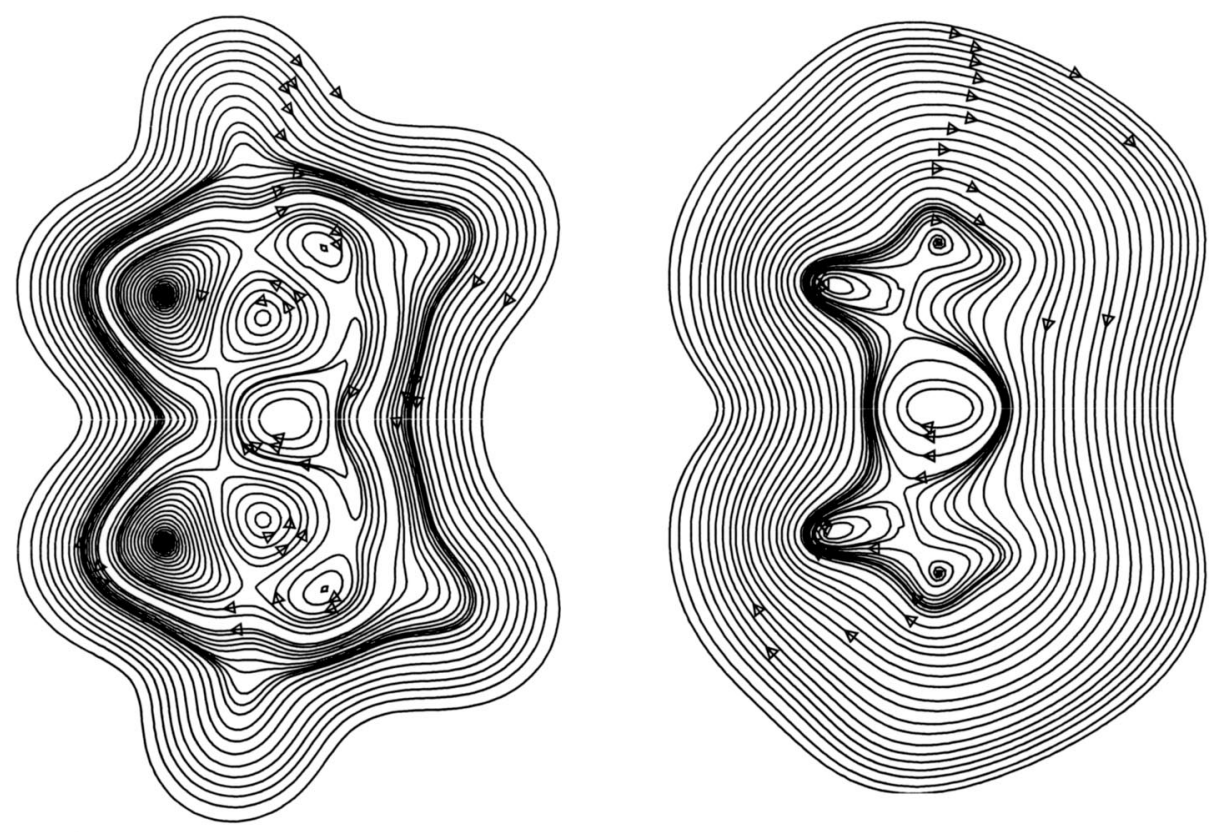

FIG. 4. Streamlines of the current density vector field in a plane parallel to that of the molecule, at the distance of $0.8 \mathrm{bohr}$, induced by a magnetic field directed perpendicular and pointing outwards. The plots on the top (bottom) are for furo[2,3- $b]$ furan (furo[3,4-c]furan). The trajectories
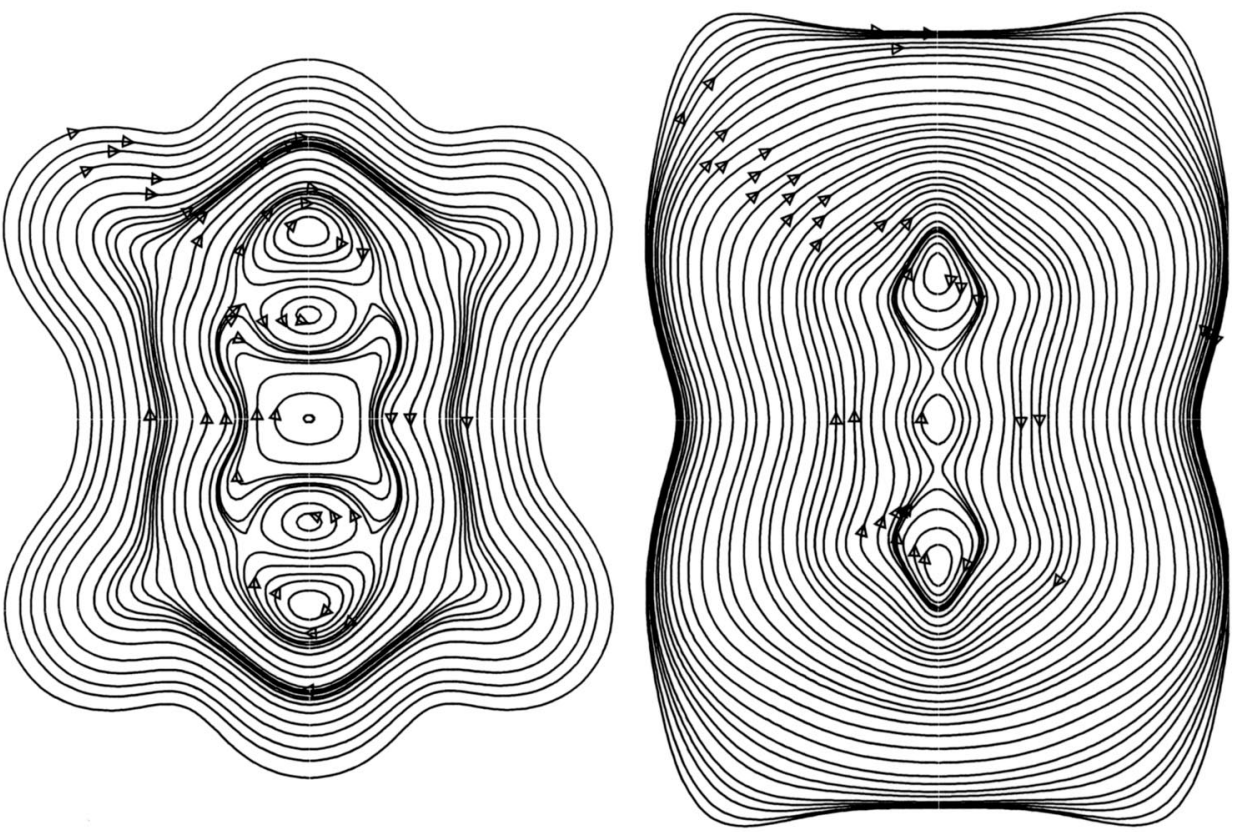
for the total $(\pi$-electron contribution) are represented on the left (right) hand side.

outermost diamagnetic vortex branches out in the proximity of the molecular ring, originating internal vortices separated by saddle-type stagnation lines.

The current regime found for the furo[3,4-c] furan is virtually identical to that observed in the corresponding pyrrolo-pyrrole isomer. ${ }^{6}$ Five vortices, centered on the longitudinal axis of the molecule, and four saddles, can be seen on the bottom left of Fig. 4. The innermost diamagnetic vortex rotating about the center of mass, over the $\mathrm{C}-\mathrm{C}$ bond connecting two fused rings, is separated from the couple of diamagnetic vortices at the opposite sides of the $x$ axis, by two intermediate paramagnetic vortices, each rotating about the center of a furan ring.

Vortices, saddles, and foci are also observed in the plot for the $[2,3-b]$ isomer on the top of Fig. 4.

A comparison of the plots for the intensity $|\mathbf{J}|=\rho|\mathbf{v}|$ and for the electron charge density $\rho$ of the unperturbed molecules give additional information on the magnetic aromaticity of furo-furans. A uniform magnetic field, normal to the molecular plane of a diatropic system, induces a stationary motion of the electronic charge. $\rho(\mathbf{r})$ is a function whose value remains the same at all points $\mathbf{r}$ in the presence of the magnetic perturbation. It shows sharp localized maxima close to carbon and oxygen nuclei. The $|\mathbf{J}(\mathbf{r})|$ modulus is a much smoother function presenting smaller oscillations among minima and maxima.

For the $\pi$ electrons in particular, which are commonly described as delocalized, the maps indicate that the intensity of the current in contiguous domains is more uniform than charge distribution. This might be related to the fact that the local Madelung-Landau-London average velocity $\mathbf{v}$ over the bonds is higher than over the nuclei. ${ }^{31}$ 


\section{CONCLUSIONS}

The present work confirms the conclusion arrived at in the first paper of this series ${ }^{6}$ that CTOCD computational schemes for magnetic susceptibilities and nuclear magnetic shieldings are practical and effective tools, alternative to those employing London orbitals.

The diatropic character of bis-heteropentalenes in the presence of a magnetic field at right angles to the molecular plane is visualized by plots for the streamlines of the electron current density and by the relative maps for the intensity of the current density vector field. Reliable ring-current models for these molecules, accounting for the essentials of electron flow in space, are now available.

A "matrix" of relative diatropicity of fused fivemembered heterocyclic rings formed by pyrrole and furan units can be constructed assuming the out-of-plane tensor component of the magnetic susceptibility, and of proton shielding of the hydrogen in position 3 with respect to the heteroatom, as quantitative descriptors. Diatropicity increases when the magnitude of the former increases and the value the latter decreases.

However, the magnetic criteria for aromaticity may conflict with other quantitative indices. The diatropicity of bisheteropentalenes does not agree with a naive aromaticity sequence based on self-consistent field (SCF) energies, which would indicate that the $[3,4-c]$ isomer is the least aromatic, even if it shows the most intense diamagnetic ring currents. In fact, the computed SCF energies from the basis set used in the calculation of magnetic properties are -379.33534 , $-379.33335,-379.26252$, and -379.32884 hartree, respectively, for (1), (2), (3), and (4), that is, the $[3,4-c]$ isomer is less stable than the others at the SCF level of accuracy. This trend has been confirmed via a B3LYP calculation.

Therefore, it should be emphasized that the "matrices" defined in this section and in Sec. II A, and scales based on increasing absolute value of the out-of-plane susceptibility component, and/or decreasing out-of-plane proton shielding, only account for relative magnetotropicity.

\section{ACKNOWLEDGMENTS}

Financial support for this work from the European research and training network "Molecular Properties and Materials (MOLPROP)," Contract No. HPRN-CT-2000-00013, from DGI (Dirección General de Investigación) of the Spanish MCyT (Ministerio de Ciencia y Tecnología) under
Project No. BQU2001-2935-C02-01, and from the Italian MURST (Ministero dell'Università e della Ricerca Scientifica e Tecnologica), via FIRB and $60 \%$ funds, is gratefully acknowledged.

${ }^{1}$ P. Lazzeretti, in Progress in Nuclear Magnetic Resonance Spectroscopy, edited by J. W. Emsley, J. Feeney, and L. H. Sutcliffe (Elsevier, Amsterdam, 2000), Vol. 36, pp. 1-88.

${ }^{2}$ J. A. N. F. Gomes and R. B. Mallion, Chem. Rev. 101, 1349 (2001).

${ }^{3}$ P. von Ragué Schleyer, Chem. Rev. 101, 1115 (2001), and articles therein.

${ }^{4}$ P. Lazzeretti, Phys. Chem. Chem. Phys. 6, 217 (2004).

${ }^{5}$ G. Subramanian, P. von Ragué Schleyer, and H. Jiao, Angew. Chem., Int. Ed. Engl. 35, 2638 (1996).

${ }^{6}$ I. G. Cuesta, R. S. Jartín, A. S. de Merás, and P. Lazzeretti, J. Chem. Phys. 119, 5518 (2003).

${ }^{7}$ F. B. van Duijneveldt, Research Report RJ 945, IBM, 1971.

${ }^{8}$ R. Zanasi and P. Lazzeretti, J. Chem. Phys. 85, 5924 (1986).

${ }^{9}$ M. J. Frisch, G. W. Trucks, H. B. Schlegel et al., GAUSSIAN 94, Revision B.3, Gaussian, Inc., Pittsburgh, PA, 1995

${ }^{10}$ P. Lazzeretti, M. Malagoli, and R. Zanasi, Research Report 1/67, CNR, 1991.

${ }^{11}$ T. A. Keith and R. F. W. Bader, Chem. Phys. Lett. 210, 223 (1993).

${ }^{12}$ T. A. Keith and R. F. W. Bader, J. Chem. Phys. 99, 3669 (1993).

${ }^{13}$ T. A. Keith and R. F. W. Bader, Can. J. Chem. 74, 185 (1996).

${ }^{14}$ R. F. W. Bader and T. A. Keith, Int. J. Quantum Chem. 60, 373 (1996).

${ }^{15}$ P. Lazzeretti, M. Malagoli, and R. Zanasi, J. Chem. Phys. 102, 9619 (1995).

${ }^{16}$ P. Lazzeretti and R. Zanasi, Int. J. Quantum Chem. 60, 249 (1996).

${ }^{17}$ R. Zanasi, J. Chem. Phys. 105, 1460 (1996).

${ }^{18}$ F. London, J. Phys. Radium 8, 397 (1937).

${ }^{19}$ A. E. Hansen and T. D. Bouman, J. Chem. Phys. 82, 5035 (1985), this is the first reference in which the reinterpretation of the GIAO acronym for gauge-including-atomic-orbitals has been proposed, see footnote 6 , p. 5047.

${ }^{20}$ T. Helgaker, H. J. A. Jensen, P. Jørgensen et al., DALTon, An electronic structure program, Release 1.2, Dalton (2001).

${ }^{21}$ P. Lazzeretti, M. Malagoli, and R. Zanasi, Chem. Phys. Lett. 220, 299 (1994).

${ }^{22}$ S. Coriani, P. Lazzeretti, M. Malagoli, and R. Zanasi, Theor. Chim. Acta 89, 181 (1994).

${ }^{23}$ R. Zanasi, P. Lazzeretti, M. Malagoli, and F. Piccinini, J. Chem. Phys. 102, 7150 (1995).

${ }^{24}$ R. Zanasi, P. Lazzeretti, and P. W. Fowler, Chem. Phys. Lett. 278, 251 (1997).

${ }^{25}$ W. H. Flygare, Chem. Rev. 74, 653 (1974).

${ }^{26}$ D. H. Sutter and W. H. Flygare, in Topics in Current Chemistry (Springer, Berlin, 1976), Vol. 63, pp. 89-196.

${ }^{27}$ J. Hoarau, N. Lumbroso, and A. Pacault, C. R. Acad. Sci. (Paris) 242, 1702 (1956).

${ }^{28}$ R. H. Mitchell, Chem. Rev. 101, 1301 (2001).

${ }^{29}$ A. Ligabue and P. Lazzeretti, J. Chem. Phys. 116, 964 (2002). See EPAPS document E-JCPSA6-115-304148. This document may be reached via the EPAPS homepage http://www.aip.org/pubservs/epaps.html

${ }^{30}$ A. Ligabue, A. Soncini, and P. Lazzeretti, J. Am. Chem. Soc. 124, 2008 (2002).

${ }^{31}$ A. Ligabue and P. Lazzeretti, J. Chem. Phys. 116, 964 (2002). 